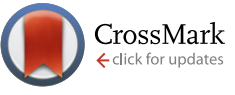

Cite this: Med. Chem. Commun., 2016, 7, 500

Received 30th October 2015 Accepted 24th December 2015

DOI: $10.1039 / c 5 m d 00499 c$

www.rsc.org/medchemcomm

\title{
Targeting the protein backbone with aryl halides: systematic comparison of halogen bonding and $\pi \cdots \pi$ interactions using $\mathbf{N}$-methylacetamide $\dagger+$
}

\author{
M. O. Zimmermann ${ }^{\text {ab }}$ and F. M. Boeckler ${ }^{\star a b}$
}

\begin{abstract}
The ubiquitous amide moiety of the protein backbone is an essential interaction partner in any binding site. Using quantum mechanical calculations, we evaluate how to target this moiety through halogen bonding. In contrast to previously employed atom-centric spherical scans, we make use of planar scans to additionally account for the delocalised $\pi$-electrons of the amide. The scans showed that perpendicular interaction geometries are moderately strong while favouring the carbonyl oxygen atom at lower distances. Gradually moving to a parallel arrangement results in a transition from $\sigma$-hole interactions toward $\pi \cdots \pi$ and dipolar interactions and higher interaction energies.
\end{abstract}

\section{Introduction}

Halogen bonding can be described as a favourable, directional interaction between an electropositive region on a halogen atom, the $\sigma$-hole,,$^{1,2}$ and a nucleophilic interaction partner such as an oxygen atom or the $\pi$-system of aromatic moieties. ${ }^{3-5}$ In the last few years various examples of successful application of halogen bonding in biological systems have been reported in the literature. ${ }^{6-11}$ With increased understanding of this rather uncommon type of molecular interaction fostering molecular design, the number of rationally designed halogen bonds in protein-ligand complexes is steadily rising.

Using quantum mechanical calculations, we have recently published systematic evaluations of different halogen bond acceptors in a protein binding site that demonstrate how to properly target certain residues through halogen bonding. These studies include the carbonyl oxygen atom of the protein backbone, ${ }^{12}$ the sulfur of methionine, ${ }^{13}$ and the unprotonated nitrogen atom in the side chain of histidine. ${ }^{14}$

Among these, the carbonyl oxygen is undoubtedly the most relevant halogen bonding acceptor. ${ }^{4,12,15}$ Being a part of an $\alpha$-helix or a $\beta$-sheet, this carbonyl oxygen atom is involved in a hydrogen bond with the amino moiety of a proximal amino acid. It has been shown that halogen atoms can still,

\footnotetext{
${ }^{a}$ Department of Pharmaceutical and Medicinal Chemistry, Institute of Pharmaceutical Sciences, Eberhard Karls Universität Tübingen, Auf der Morgenstelle 8, 72076 Tübingen, Germany. E-mail: frank.boeckler@uni-tuebingen.de ${ }^{b}$ Center for Bioinformatics Tuebingen (ZBIT), Eberhard Karls University Tuebingen, Sand 1, 72076 Tuebingen, Germany

$\dagger$ The authors declare no competing interests.

\$ Electronic supplementary information (ESI) available. See DOI: 10.1039/ c5md00499c
}

orthogonally, target this oxygen atom without significant loss of interaction energy ${ }^{16}$ The carbonyl oxygen atom, however, is not the sole possible interaction partner of the protein backbone. Crystal structures have shown that the amide group is planar with only very small deviations from a dihedral angle of $180^{\circ}$ being tolerated. This means, that $\pi$-electrons are delocalised across the three heavy atoms of this moiety, making the whole amide function a possible halogen bonding acceptor.

A recent scan of the $\mathrm{PDB}^{17}$ (as of July 2015) for halogen bonds towards polar protein atoms yielded 204 contacts (in 133 unique PDB codes) between chlorine and the nitrogen atom of the backbone with good $\sigma$-hole angles of $\geq 160^{\circ}$ in distances between 3.0 and $4.5 \AA$ A. For bromine this number is reduced to 53 hits ( 26 unique) while for iodine only 29 presumably good contacts (18 unique) have been found. Fig. 1 shows two halogen bonding examples with the amide moiety of the protein backbone that were found through the scan. In both examples the $\sigma$-hole angles between the halogen atoms
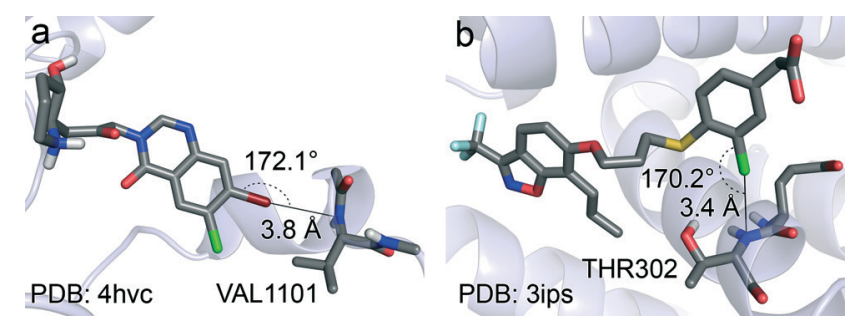

Fig. 1 Halogen bonds addressing the amide moiety of the protein backbone. (a) Bromine halogen bond addressing the amide function of VAL1101 in human prolyl-tRNA synthetase (PDB: $4 \mathrm{hvc}^{18}$ ). (b) Chlorine halogen bond with the amide function of THR302 in LXR- $\alpha$ (PDB: $3 \operatorname{ips}^{19}$ ). 
and the nitrogen atom of the protein backbone were the highest among all involved atoms, indicating that the $\mathrm{C}$ $\mathrm{X} \cdots \mathrm{N}$ contact is preferred over the $\mathrm{C}-\mathrm{X} \cdots \mathrm{O}$ contact. In human prolyl-tRNA synthetase (Fig. 1a), the co-crystallised ligand halofuginone forms a bromine halogen bond with the amide moiety of VAL1101 (PDB: 4hvc). ${ }^{18}$ The $\sigma$-hole angle to the nitrogen atom is with $172.1^{\circ}$ almost optimal. The $\sigma$-hole angle to the carbonyl oxygen atom is with $145.1^{\circ}$ rather suboptimal and shows that the backbone nitrogen atom should not be neglected in halogen bond evaluations.

Fig. $1 \mathrm{~b}$ shows the ligand $\mathrm{F}_{3}$ methylAA bound to the protein LXR- $\alpha$ engaging in a chlorine halogen bond with the backbone nitrogen atom of THR302 (PDB: 3ips). ${ }^{19}$ The $\sigma$-hole angle is $170.2^{\circ}$ and the interaction distance is $3.4 \AA$. Here, the $\sigma$-hole angle to the carbonyl oxygen atom is with $152.5^{\circ}$ only moderate.

\section{Results and discussion}

To systematically analyse the targetability of the protein amide moiety on the basis of quantum mechanical calculations, we used MP2/TZVPP-optimised structures of $N$-methylacetamide, serving as a model system for the protein backbone, and iodobenzene as the halogen bond donor to set up planar scans of the protein backbone. The setup of the scan can be seen in Fig. 2. Initially, the iodobenzene molecule is positioned orthogonally to the plane of the amide moiety of $\mathrm{N}$-methylacetamide ( $\mathrm{XZ}$-plane) in the desired interaction distance and orientation (Fig. 2a). By moving the ligand in both directions ( $X$ and $Z$ ) of the plane in steps of $0.25 \AA$ (Fig. 2b), 1320 different interaction geometries are generated (Fig. 2c). At all times, the distance to the plane of the amide moiety and the orientation of the aromatic ring remain unaltered.

For each of the distances 2.75, 3.0, 3.25, 3.5, 3.75, and 4.0 $\AA$ these 1320 geometries are calculated as TPSS(D3)/TZVPP single points and the adduct formation energy (in $\mathrm{kJ} \mathrm{mol}^{-1}$ ) is determined. The visualisation of all six plane scans between $\mathrm{N}$-methylacetamide and iodobenzene can be seen in Fig. 3a. Each square in the depicted 2D-plots represents the position of the ligand's iodine atom in the stated distance above the plane of the amide moiety. Energy values $-10 \mathrm{~kJ}$ $\mathrm{mol}^{-1}$ are highlighted with a black border. As expected, scan
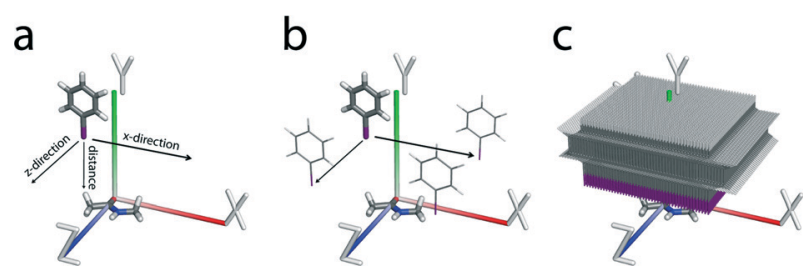

Fig. 2 Setup of the plane scan. (a) Starting orientation of $\mathrm{N}$-methylacetamide and iodobenzene. Scan directions are depicted using black arrows. (b) Dimensions of the plane scan of the desired interaction distance. (c) All interaction geometries for one plane scan and one distance. positions above the oxygen atom belong to the most favourable interaction geometries. Increasing the halogen bond distance to the plane of the amide moiety to $d \geq 3.5 \AA$ shows an extension of the preferred interaction geometries, also including positions above the whole amide function, indicating improved $\sigma$-hole $\cdots \pi$ contacts.

An additional reason for this behaviour could be that short repulsive contacts (indicated by uncoloured data points in Fig. 3a) to the methyl groups of $N$-methylacetamide are alleviated at larger distances. In a regular protein structure, of course, the size and orientation of the side chains will determine, whether a perpendicular interaction as investigated herein, is feasible. Overall, the best interaction energy is found at a distance of $3.25 \AA$ with $-14.9 \mathrm{~kJ} \mathrm{~mol}^{-1}$. At interaction distances of $4.0 \AA$ and higher the most favourable energy does not exceed $-10 \mathrm{~kJ} \mathrm{~mol}{ }^{-1}$. These scans indicate that, apart from the carbonyl oxygen atom, the whole amide moiety is still a moderately good halogen bond acceptor. For comparative purposes, Fig. 3b shows oxygen-centric spherical scans between iodobenzene and $N$-methylacetamide which have been calculated using MP2/TZVPP. ${ }^{12,15}$ The colour scale has been adapted to match with the plane scans. The spherical scan at $3.0 \AA$ shows a large connected area of highly favourable interaction geometries around the carbonyl oxygen atom. At a higher distance of $3.8 \AA$ the most favourable geometries are found perpendicular to the amide moiety.

Despite the possibility of small systemic shifts between absolute interaction energies in the comparison of MP2/TZVPP and TPSS(D3)/TZVPP calculations, a high semi-quantitative similarity is obvious. Thus, the possibility of alternative $\sigma$-hole $\cdots \pi$ contacts already detectable in the spherical scans at larger distances is systematically confirmed in the plane scans. For comparison, we have added the plane scans for chlorobenzene and bromobenzene in distances of 3.0, 3.5, and 4.0 A to the ESIt (Fig. S1). For the lighter halogens, the interaction energy is significantly decreased. This is in line with previous observations. ${ }^{12}$

As a next step, the interaction angle of iodobenzene to the plane of the amide moiety is altered from $0^{\circ}$ to $90^{\circ}$ in steps of $15^{\circ}$ to evaluate the transition from $\sigma$-hole $\cdots \pi$ interactions to $\pi \cdots \pi$-interactions. The setup of the scan is similar to that described in Fig. 2. Prior to the generation of the input geometries for the calculations, the benzene ring is tilted by $15^{\circ}$, $30^{\circ}, 45^{\circ}, 60^{\circ}, 75^{\circ}$, or $90^{\circ}$. The position of the iodine atom remains unchanged compared to the previous scans, however, the exposure of the $\sigma$-hole toward the $\pi$-electron density is steadily decreased. To focus on the best interactions with the least clashes, the tilted plane scans were only performed for a distance of $3.5 \AA$ A. Here, 1412 geometries per tilt angle are generated and TPSS(D3)/TZVPP single points are calculated (Fig. 4). Each coloured square corresponds to the position of the iodine of iodobenzene at a distance of $3.5 \AA$ to the plane of the amide moiety, while the benzene ring is gradually tilted towards the plane. At tilt angles of $15^{\circ}$ and $30^{\circ}$, the best interaction geometries are found around the carbonyl oxygen atom. At these angles, the $\sigma$-hole interaction still represents 
a $2.75 \AA$



$3.50 \AA$

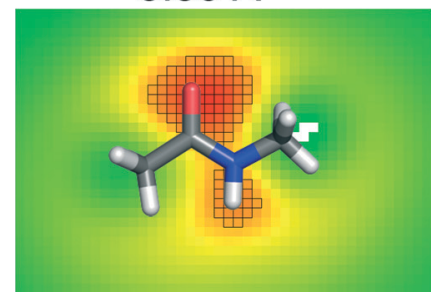

$3.00 \AA$

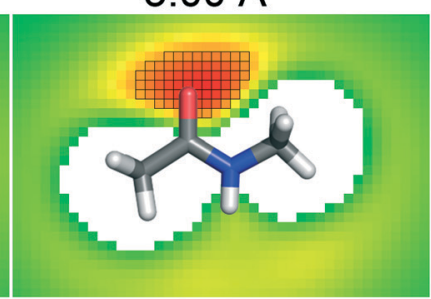

$3.75 \AA$

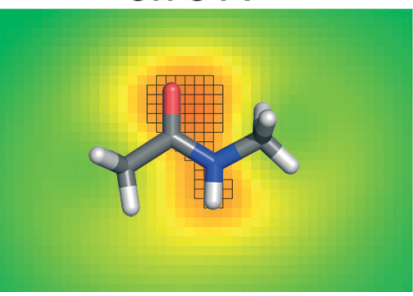

$3.25 \AA$

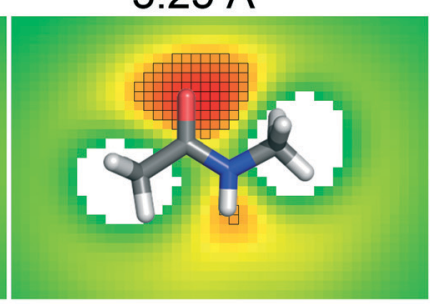

$4.00 \AA$

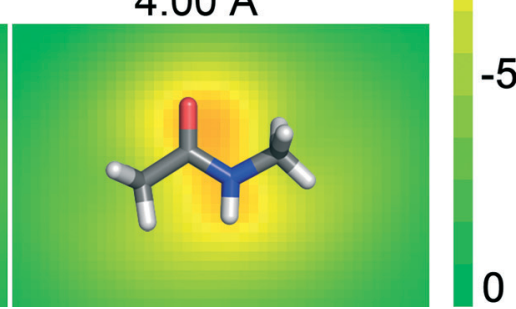

b
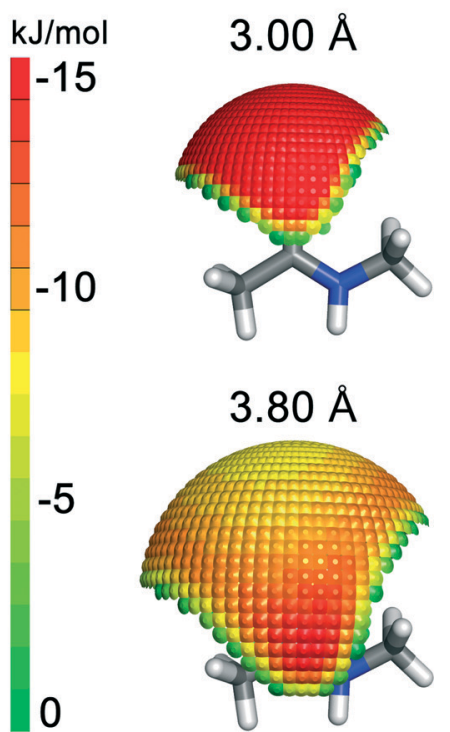

Fig. 3 Evaluation of the halogen bonding interaction between iodobenzene and $N$-methylacetamide. Positive energy values $\left(>0 \mathrm{~kJ}\right.$ mol $\left.{ }^{-1}\right)$ are not shown. (a) Plane scans at six different interaction distances (2.75-4.0 Å) calculated using TPSS(D3)/TZVPP. (b) Spherical scans around the carbonyl oxygen atom calculated using MP2/TZVPP.

the main contribution to the overall interaction energy. At tilt angles of $45^{\circ}$ and higher, a transition towards the lower part of the plane is observed and the hotspot around the carbonyl oxygen vanishes. This is in line with observations in other systematic studies, clearly indicating that deviations of the $\sigma$-hole angle by more than $45^{\circ}$ from the optimum of $180^{\circ}$ is highly detrimental for the halogen bond contribution to the overall binding energy. ${ }^{12-14}$ At a tilt angle of $90^{\circ}$, generating a shifted parallel $\pi$-stacking arrangement of iodobenzene and

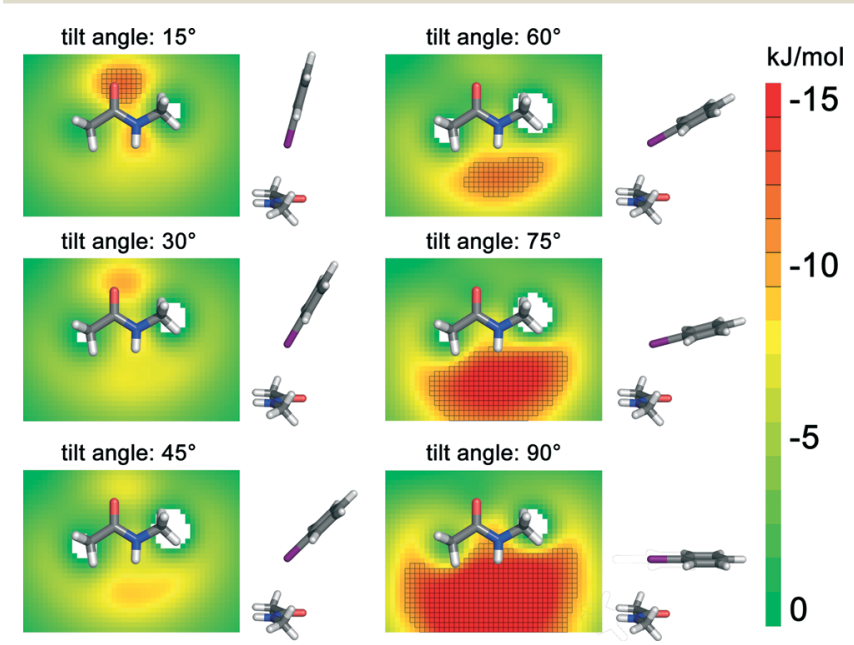

Fig. 4 Tilted plane scans of $\mathrm{N}$-methylacetamide and iodobenzene for tilt angles $15^{\circ}, 30^{\circ}, 45^{\circ}, 60^{\circ}, 75^{\circ}$, and $90^{\circ}$. As a reference, for each plane scan the respective tilt angle is depicted (to the right of each plot) by one selected interaction geometry between $\mathrm{N}$-methylacetamide and iodobenzene. The colour scale is similar to that shown in Fig. 3. Positive energy values $\left(>0 \mathrm{~kJ} \mathrm{~mol}^{-1}\right)$ are not shown.
$N$-methylacetamide, the highest interaction energy between the two molecules is roughly $-20 \mathrm{~kJ} \mathrm{~mol}^{-1}$. In comparison, the best interaction geometry of the oxygen-centric spherical scan between iodobenzene and $N$-methylacetamide at $3.0 \AA$ (see Fig. $3 \mathrm{~b}$ ) is $-14.1 \mathrm{~kJ} \mathrm{~mol}^{-1}$ using TPSS(D3)/TZVPP.

Besides $\pi \cdots \pi$ and $\mathrm{CH}^{\cdots} \cdots \pi$ interactions, it should be noted that the dipole moments of both molecules are aligned in an antiparallel manner, when the iodine atom is located within the broad red hotspot below $N$-methylacetamide. Thus, this attractive dipole...dipole interaction can also provide a significant contribution to the observed energies. Tilted plane scans for chlorobenzene and bromobenzene can be found in the ESIt (Fig. S2). At tilt angles of $45^{\circ}$ and (even more) $90^{\circ}$, the distribution of interaction energies of all three halobenzenes converges to a similar pattern.

In order to better understand these 2D-plots, Fig. 5 shows the interaction geometries of two selected examples. The example taken from the plane scan with a tilt angle of $30^{\circ}$ shows a moderately good halogen bonding geometry. The interaction energy amounts to $-10.0 \mathrm{~kJ} \mathrm{~mol}^{-1}$. The halogen bond is directed towards the $\mathrm{C}=\mathrm{O}$ double bond of the carbonyl moiety. The second example is taken from the plane scan with a tilt angle of $90^{\circ}$. The high interaction energy of $-19.6 \mathrm{~kJ} \mathrm{~mol}^{-1}$ most certainly arises from $\pi$-interactions or antiparallel dipolar interactions between the benzene ring and $N$-methylacetamide. The tilted plane scans showed that the transition from halogen bonding to $\pi$-interactions is seamless. A cutoff cannot easily be determined. This can be especially difficult when it comes to the creation of a scoring function for this type of interaction. Important or even crucial limitations of these parallel interactions are steric clashes based on $\varphi-/ \psi$-angles of the 


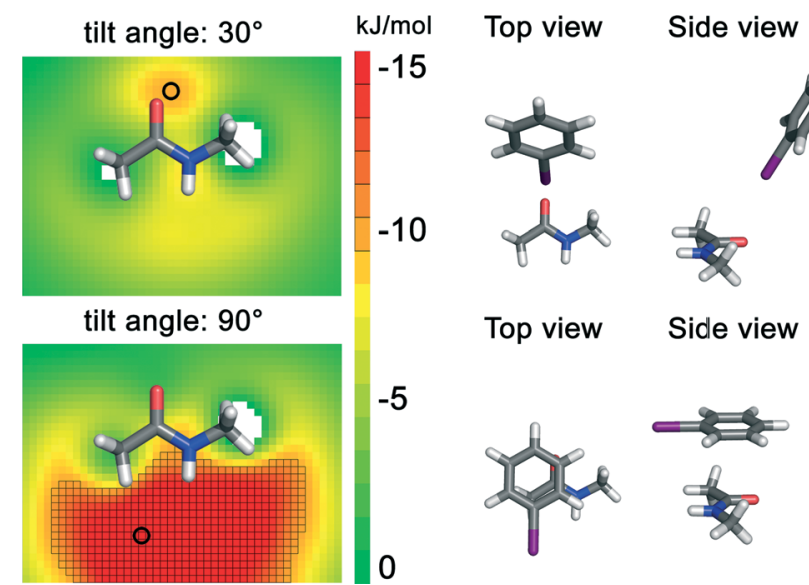

Fig. 5 Selected examples of the titled plane scans. For the tilt angles $30^{\circ}$ and $90^{\circ}$ the highlighted geometry (black circle) is shown from the side view and top view.

protein backbone, when involved in specific secondary structure, as well as based on the size and orientation of the amino acid side chains.

In order to scrutinise the plausibility of such close (anti) parallel $\pi$-interactions, we have modified our PDB scan to highlight binding situations, where in spite of the possibility to form a halogen bond, the $\pi \cdots \pi$ interaction is preferred.

We limited the angle between the planes to $\leq 20^{\circ}$ and the distance between the centre of mass of the aryl moiety and any heavy atom of the amide moiety to $4.5 \AA$. For chlorine, 536 contacts were found in 312 unique crystal structures.

Similar to the results obtained for the halogen bonding analysis, for bromine ( 80 in total, 43 unique) and iodine (35 in total, 18 unique) significantly less contacts were found.

Four selected examples of the PDB scan are shown in Fig. 6. Bound to purine nucleoside phosphorylase, the cocrystalised ligand 8-iodo-guanine forms $\pi \cdots \pi$ contacts with the amide moiety of CYS136 and GLY137 in an interaction distance of 4.0-4.1 Å. Fig. 6b shows 4-bromomethoxyphenol bound to factor VIIa. The ligand forms favourable $\pi \cdots \pi$ contacts with the amide of TRP215 and GLY216. Due to a decreasing van der Waals radius, for bromine, and even more pronounced for chlorine, much closer $\pi \cdots \pi$ contacts were found in the PDB scan for ligands containing the lighter halogens. In Fig. 6c, 3,4-dichlorobenzoate interacts with the amide moiety of GLY305 and THR306 at distances between 3.3 and $3.7 \AA$ to the centre of mass of the ligand's aromatic ring. Bound to factor Xa, the ligand M55113 engages in a chlorine halogen bond with the aromatic side chain of TYR228 (Fig. 6d). This contact is considered to be of great importance for high ligand affinity. ${ }^{23}$ Probably of similar importance is that the ligand is sandwiched between the amide moiety of TRP215 and GLY216 in distances between 3.7 and 3.9 $\AA$, and the amide moiety of ALA190 and CYS191 at larger interaction distances of $4.5 \AA$.

Due to sterical reasons, the amide moieties of amino acids with smaller side chains, such as alanine and glycine, are

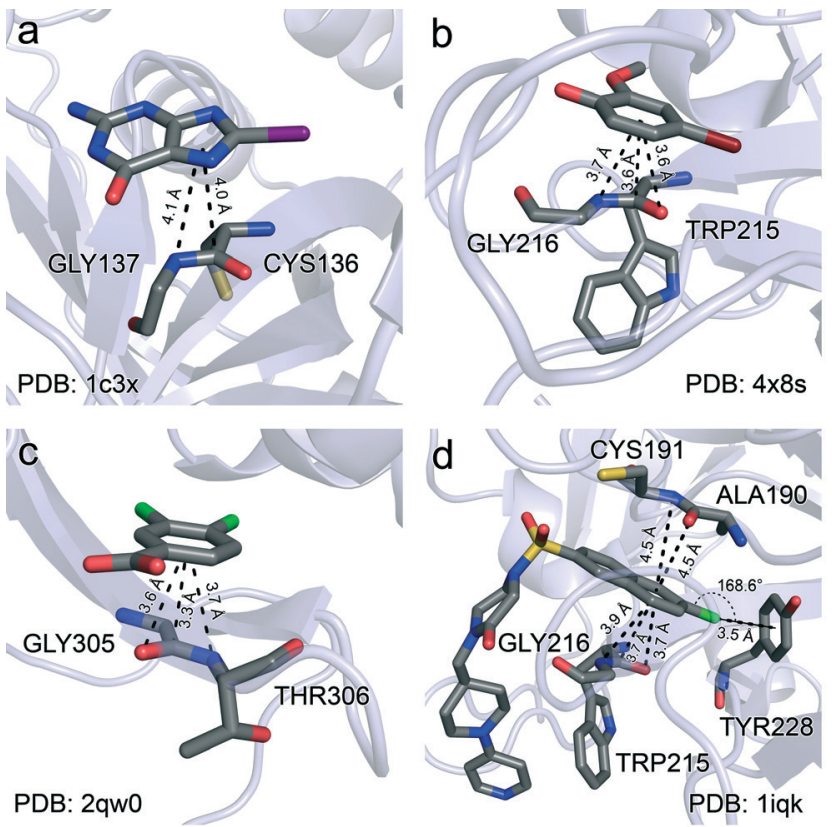

Fig. 6 Selected examples for $\pi \cdots \pi$ interactions: (a) 8-lodo-guanine interacting with the amide moiety of CYS136 and GLY137 (PDB: $\left.1 \mathrm{c} 3 \mathrm{x}^{20}\right)$, (b) 4-bromo-methoxyphenol interacting with the amide moiety of TRP215 and GLY216 (PDB: $4 \times 8 \mathrm{~s}^{21}$ ), (c) 3,4-dichlorobenzoate interacting with the amide moiety of GLY305 and THR306 (PDB: 2qw0 (ref. 22)), (d) M55113 forming a chlorine halogen bond with the aromatic side chain of TYR228. Additionally, the ligand is sandwiched between the amide moieties of ALA190 and CYS191, as well as TRP215 and GLY216 (PDB: 1iqk).

obviously most suitable to engage frequently in close $\pi \cdots \pi$ contacts.

\section{Methods}

\section{Structure optimisations and spherical scans}

Geometry optimisations and spherical scans for the halobenzenes and $N$-methylacetamide were carried out at the MP2/TZVPP ${ }^{24}$ level of theory using the TURBOMOLE $6.4^{25,26}$ suite of programs. Relativistic effects for iodine were considered by an effective core potential (ECP). ${ }^{27}$ The calculations were done in combination with the resolution of identity (RI) technique $^{28-30}$ and the frozen core approximation. The frozen core orbitals were attributed by the default setting in TURBOMOLE by which all orbitals possessing energies below 3.0 au are considered core orbitals. The SCF convergence criterion was increased to $10^{-8}$ Hartree for all calculations.

\section{DFT single point calculations}

All TPSS(D3) calculations were carried out using the TURBOMOLE $6.4^{25,26}$ suite of programs. The employed basis sets was of triple- $\zeta$ quality (def2-TZVPP). ${ }^{24}$ Calculations were performed using the RI approximation ${ }^{31-33}$ and the TPSS $^{34}$ functional. The functional was augmented with the third version of Grimme's empirical dispersion correction, ${ }^{35,36}$ 
which is indicated by adding a "(D3)" to the name, i.e. TPSS(D3).

\section{Conclusions}

The herein presented evaluation of the protein backbone amide function through planar scans revealed that the whole moiety has the potential to be targeted through $\sigma$-hole bonding. While the carbonyl oxygen atom is the most relevant and potent acceptor, the delocalized $\pi$-electrons of the amide can be targeted, particularly at distances larger than $3.5 \AA$. When tilted from a perpendicular to a (anti)parallel arrangement, a seamless transition from $\sigma$-hole interactions to $\pi \cdots \pi$-stacking and antiparallel dipolar interactions is observed. In comparison to the latter, the $\sigma$-hole bonds are less compromised by side chain orientation or secondary structure of the protein backbone.

Key findings:

- A transition from $\sigma$-hole interactions involving n-electrons of the carbonyl function to $\sigma$-hole interactions with the $\pi$-electrons occurs starting from $3.5 \AA$.

- The halogen bond quality decreases with increasing tilt angles and the interaction vanishes above $45^{\circ}$.

- Above tilt angles of $60^{\circ}$, the $\pi \cdots \pi$ contact between the aryl moiety and the amide can surpass the quality of the optimal $\sigma$-hole interaction in certain geometries.

\section{Acknowledgements}

High performance computing resources of the BW-grid were kindly made available by the federal state of BadenWuerttemberg. This research was supported in part by the bwHPC initiative and the bwHPC-C5 project provided through associated compute services of the JUSTUS HPC facility at the University of Ulm. bwHPC and bwHPC-C5 (http://www.bwhpcc5.de) are funded by the Ministry of Science, Research and the Arts Baden-Wuerttemberg (MWK) and the Germany Research Foundation (DFG).

\section{Notes and references}

1 T. Clark, M. Hennemann, J. S. Murray and P. Politzer, J. Mol. Model., 2007, 13, 291-296.

2 P. Politzer, J. S. Murray and T. Clark, Phys. Chem. Chem. Phys., 2013, 15, 11178-11189.

3 G. R. Desiraju, P. S. Ho, L. Kloo, A. C. Legon, R. Marquardt, P. Metrangolo, P. Politzer, G. Resnati and K. Rissanen, Pure Appl. Chem., 2013, 85, 1711-1713.

4 R. Wilcken, M. O. Zimmermann, A. Lange, A. C. Joerger and F. M. Boeckler, J. Med. Chem., 2013, 56, 1363-1388.

5 M. O. Zimmermann, A. Lange, R. Wilcken, M. B. Cieslik, T. E. Exner, A. C. Joerger, P. Koch and F. M. Boeckler, Future Med. Chem., 2014, 6, 617-639.

6 A. R. Voth and P. S. Ho, Curr. Top. Med. Chem., 2007, 7, 1336-1348.

7 O. Fedorov, K. Huber, A. Eisenreich, P. Filippakopoulos, O. King, A. N. Bullock, D. Szklarczyk, L. J. Jensen, D. Fabbro, J.
Trappe, U. Rauch, F. Bracher and S. Knapp, Chem. Biol., 2011, 18, 67-76.

8 L. A. Hardegger, B. Kuhn, B. Spinnler, L. Anselm, R. Ecabert, M. Stihle, B. Gsell, R. Thoma, J. Diez, J. Benz, J.-M. Plancher, G. Hartmann, Y. Isshiki, K. Morikami, N. Shimma, W. Haap, D. W. Banner and F. Diederich, ChemMedChem, 2011, 6, 2048-2054.

9 J. Fanfrlik, M. Kolar, M. Kamlar, D. Hurny, F. X. Ruiz, A. Cousido-Siah, A. Mitschler, J. Rezac, E. Munusamy, M. Lepsik, P. Matejicek, J. Vesely, A. Podjarny and P. Hobza, ACS Chem. Biol., 2013, 8, 2484-2492.

10 J. Fanfrlík, F. X. Ruiz, A. Kadlčíková, J. Řezáč, A. Cousido-Siah, A. Mitschler, S. Haldar, M. Lepšík, M. H. Kolář, P. Majer, A. D. Podjarny and P. Hobza, ACS Chem. Biol., 2015, 10, 1637-1642.

11 R. Wilcken, X. Liu, M. O. Zimmermann, T. J. Rutherford, A. R. Fersht, A. C. Joerger and F. M. Boeckler, J. Am. Chem. Soc., 2012, 134, 6810-6818.

12 R. Wilcken, M. O. Zimmermann, A. Lange, S. Zahn and F. M. Boeckler, J. Comput.-Aided Mol. Des., 2012, 26, 935-945.

13 R. Wilcken, M. O. Zimmermann, A. Lange, S. Zahn, B. Kirchner and F. M. Boeckler, J. Chem. Theory Comput, 2011, 7, 2307-2315.

14 A. Lange, M. O. Zimmermann, R. Wilcken, S. Zahn and F. M. Boeckler, J. Chem. Inf. Model., 2013, 53, 3178-3189.

15 M. O. Zimmermann, A. Lange and F. M. Boeckler, J. Chem. Inf. Model., 2015, 55, 687-699.

16 A. R. Voth, P. Khuu, K. Oishi and P. S. Ho, Nat. Chem., 2009, 1, 74-79.

17 F. C. Bernstein, T. F. Koetzle, G. J. Williams, E. E. Meyer Jr., M. D. Brice, J. R. Rodgers, O. Kennard, T. Shimanouchi and M. Tasumi, J. Mol. Biol., 1977, 112, 535.

18 H. Zhou, L. Sun, X.-L. Yang and P. Schimmel, Nature, 2013, 494, 121-124.

19 X. Fradera, D. Vu, O. Nimz, R. Skene, D. Hosfield, R. Wynands, A. J. Cooke, A. Haunsø, A. King, D. J. Bennett, R. McGuire and J. C. M. Uitdehaag, J. Mol. Biol., 2010, 399, 120-132.

20 J. Tebbe, A. Bzowska, B. Wielgus-Kutrowska, W. Schröder, Z. Kazimierczuk, D. Shugar, W. Saenger and G. Koellner, J. Mol. Biol., 1999, 294, 1239-1255.

21 D. L. Cheney, J. M. Bozarth, W. J. Metzler, P. E. Morin, L. Mueller, J. A. Newitt, A. H. Nirschl, A. R. Rendina, J. K. Tamura, A. Wei, X. Wen, N. R. Wurtz, D. A. Seiffert, R. R. Wexler and E. S. Priestley, J. Med. Chem., 2015, 58, 2799-2808.

22 R. Wu, A. S. Reger, J. Cao, A. M. Gulick and D. DunawayMariano, Biochemistry, 2007, 46, 14487-14499.

23 S. Roehrig, A. Straub, J. Pohlmann, T. Lampe, J. Pernerstorfer, K.-H. Schlemmer, P. Reinemer and E. Perzborn, J. Med. Chem., 2005, 48, 5900-5908.

24 F. Weigend and R. Ahlrichs, Phys. Chem. Chem. Phys., 2005, 7, 3297-3305.

25 R. Ahlrichs, M. Bar, M. Haser, H. Horn and C. Kolmel, Chem. Phys. Lett., 1989, 162, 165-169.

26 TURBOMOLE, version 6.4, TURBOMOLE GmbH, Karlsruhe, Germany, 2010. 
27 K. A. Peterson, D. Figgen, E. Goll, H. Stoll and M. Dolg, J. Chem. Phys., 2003, 119, 11113-11123.

28 M. Feyereisen, G. Fitzgerald and A. Komornicki, Chem. Phys. Lett., 1993, 208, 359-363.

29 F. Weigend, M. Haser, H. Patzelt and R. Ahlrichs, Chem. Phys. Lett., 1998, 294, 143-152.

30 C. Hattig, Phys. Chem. Chem. Phys., 2005, 7, 59-66.

31 E. J. Baerends, D. E. Ellis and P. Ros, Chem. Phys., 1973, 2, 41-51.
32 B. I. Dunlap, J. W. D. Connolly and J. R. Sabin, J. Chem. Phys., 1979, 71, 3396-3402.

33 F. Weigend, Phys. Chem. Chem. Phys., 2006, 8, 1057-1065.

34 V. N. Staroverov, G. E. Scuseria, J. Tao and J. P. Perdew, J. Chem. Phys., 2003, 119, 12129-12137.

35 S. Grimme, J. Comput. Chem., 2006, 27, 1787-1799.

36 S. Grimme, J. Antony, S. Ehrlich and H. Krieg, J. Chem. Phys., 2010, 132, 154104. 